\title{
Quantification of the UPDRS Rigidity Scale
}

\author{
Susan K. Patrick, Allen A. Denington, Michel J. A. Gauthier, Associate Member, IEEE, \\ Deborah M. Gillard, Member, IEEE, and Arthur Prochazka, Senior Member, IEEE
}

\begin{abstract}
In the clinical setting, parkinsonian rigidity is assessed using subjective rating scales such as that of the Unified Parkinson's Disease Rating System (UPDRS). However, such scales are susceptible to problems of sensitivity and reliability. Here, we evaluate the reliability and validity of a device designed to quantify parkinsonian rigidity at the elbow and the wrist. The method essentially quantifies the clinical examination and employs small sensors to monitor forces and angular displacements imposed by the clinician onto the limb segment distal to the joint being evaluated. Force and displacement data are used to calculate elastic and viscous stiffnesses and their vectorial sum, mechanical impedance. Interexaminer agreement of measures of mechanical impedance in subjects with Parkinson's disease was comparable to that of clinical UPDRS scores. Examiners tended to overrate rigidity on the UPDRS scale during reinforcement manoeuvres. Mechanical impedance was nonlinearly related to UPDRS ratings of rigidity at the elbow and wrist; characterization of such relationships allows interpretation of impedance measurements in terms of the clinical rating scales.
\end{abstract}

Index Terms-Biomedical measurements, clinical assessment, force measurement, gyroscope, limb rigidity, Parkinson's diesease, parameter estimation, reliability testing.

\section{INTRODUCTION}

$\mathbf{T}$ REATMENT of Parkinson's disease can be challenging because of variation in symptoms between patients, progression of the disease, and changing responses to medications [1]-[3]. The cardinal symptoms of Parkinson's disease are rigidity, tremor, bradykinesia, and postural instability. Rigidity responds well to levodopa and is one of the parameters monitored to evaluate the efficacy of pharmacological and surgical treatments. However, there is currently no standardized objective method of measuring rigidity. At present, the clinician manipulates the limb of the patient and rates the evoked stiffness according to an ordinal rating scale such as that of the Unified Parkinson's Disease Rating System (UPDRS, Table I). However, the subjective nature of such scales makes them open to the interpretation of the examiner. Studies of the UPDRS have found interrater reliability of the rigidity component to be "excellent" [4], "very good" [2], and "moderate" [5]. Studies of the Columbia University rating scale (on which the motor component of the UPDRS is based), the Webster rating scale, or a recently developed custom scale [6] have reported poorer

Manuscript received November 29, 1999; revised July 17, 2000 and December 8,2000 . This work was supported by the Alberta Heritage Foundation for Medical Research, the Alberta Paraplegic Foundation, the Canadian MRC, and a University of Alberta Dissertation Fellowship. A commercial party with a direct financial interest in the results of the research supporting this article has conferred, or will confer, a benefit upon one or more of the authors.

The authors are with the Division of Neuroscience, University of Alberta, Edmonton, AB T6G 2S2, Canada (e-mail: spatric@emory.edu).

Publisher Item Identifier S 1534-4320(01)01782-X.
TABLE I

UPDRS RIGIDITY RATING SCALE (ADAPTED FROM [1])

$0 \quad$ Rigidity absent

1 Rigidity slight or detectable only when activated by mirror or other movements

2 Rigidity mild to moderate

3 Rigidity marked, but full range of motion easily achieved

$4 \quad$ Rigidity severe; range of motion achieved with difficulty

levels of interrater agreement, which in some cases were no better than would be expected by chance [6]-[8]. Our own observations have shown disagreement between clinicians as to the effectiveness of medication [9], which may in fact be explained by the tendency of different raters to concentrate to different extents on the minimum, mean, or maximum rigidity evoked. It has also been recognized that the severity of one symptom may affect the assessment of another [unpublished observations], [1], [10]. A need for more precise methods has often been expressed [10]-[13].

Several groups have recognized the shortcomings of the subjective methods of assessment of rigidity and have developed methods of quantifying, among other parameters, the work involved in moving a limb [14]-[16], or the stiffness [17], [18], resonant frequency [19], or activation-induced increases in stiffness [20], [21] of the limb. Several of the earlier methods involved the use of torque motors which put unnatural constraints on movement of the limb. Some of the more recent measurement devices were designed to be used in ways that more closely resemble a clinical examination [21], [22]. The most often quoted reasons for not introducing objective methods of quantification into the clinical setting are the expense, complexity, and time involved [1].

We recently introduced a device for the quantification of limb stiffness which we applied to the measurement of parkinsonian rigidity at the elbow [9]. This device is based on the method of assessment of rigidity used routinely in the clinical setting, that is, the passive manipulation of the joint in question by the examiner. Sensors are used to monitor the force imposed by the examiner in moving the limb as well as the amount of movement, and the amount of rigidity is described in terms of mechanical impedance as calculated from these two parameters. The device is inexpensive, simple to use, and allows the examiner to feel and 
thus qualitatively rate the evoked rigidity while it is also being quantified. We found that a 50 -second trial length provided a more consistent estimate of mean stiffness than the customary clinical exam, which typically might last about $10 \mathrm{~s}$.

Any method of assessment of clinical symptoms must be valid, sensitive to changes in the level of the symptom, and reliable [2]. Since its introduction, the quantification device has undergone substantial changes to the hardware. In this paper, we test the sensors currently used, validate the algorithm used to calculate stiffness, and assess reliability of the overall approach. Since clinicians are accustomed to ordinal rating scales, it may be preferable to express quantitative measures in terms of such ratings. In this paper, we describe mathematically the relationship between quantified stiffness measures and UPDRS rigidity scores for the elbow and wrist and discuss the appropriateness of using the resulting equations to express quantified measures in terms of the UPDRS rating scale.

Parts of this paper have been presented elsewhere and appear in abstract form [23]-[25].

\section{METHODS}

\section{A. Stiffness Quantification Device}

The device was originally designed to measure parkinsonian rigidity at the elbow, and the original version has been described in detail elsewhere [9]. A more recent version is reviewed here.

To quantify rigidity of a particular joint, the examiner repeatedly flexes and extends the joint as in a regular clinical examination. The movements are imposed through two air-filled pads held distal to the joint (Fig. 1). The pads are connected to a differential force transducer (Motorola MPX10-DP) which measures the resultant force applied to the pads. A solid state piezoelectric gyroscope (Murata ENC05E) mounted on one of the force pads monitors the imposed angular velocity from which is computed displacement. (In the original version of the device, a long elastic strain gauge was used to monitor displacement; the gyroscope proved less cumbersome.) Both signals are fed into a data acquisition box where they are filtered $(9.6 \mathrm{~Hz}$ second order low-pass Sallen-key), and digitized. A Motorola $68 \mathrm{HC} 11$ microprocessor samples the signals at $20 \mathrm{~s}^{-1}$ and sends the result to a laptop computer. Alternatively, the filtered analog signals can be obtained directly from the box for external display or sampling. In addition, a keypad on the box allows input of clinical ratings or event markers.

Data collection was controlled via a user interface written in Visual Basic (Microsoft). This program invokes MATLAB (Version 4.2c.1, The MathWorks, Inc.) to perform the analysis. (A more recent, commercially available version of the device uses equivalent custom-written subroutines.) The first $0.5 \mathrm{~s}$ of each trial, with the limb held still, allows the gyroscope signal zero-velocity offset to be determined. The first four seconds of all signals are discarded to avoid analyzing data collected before the rigidity testing is actually underway. The gyroscope signal is integrated using Euler's method and detrended. Calibration of the raw data signals is then performed using linear interpolation. Calibration values are obtained by applying a $1 \mathrm{~kg}$ weight to each force pad individually, and by rotating the gyroscope through 90 degrees.

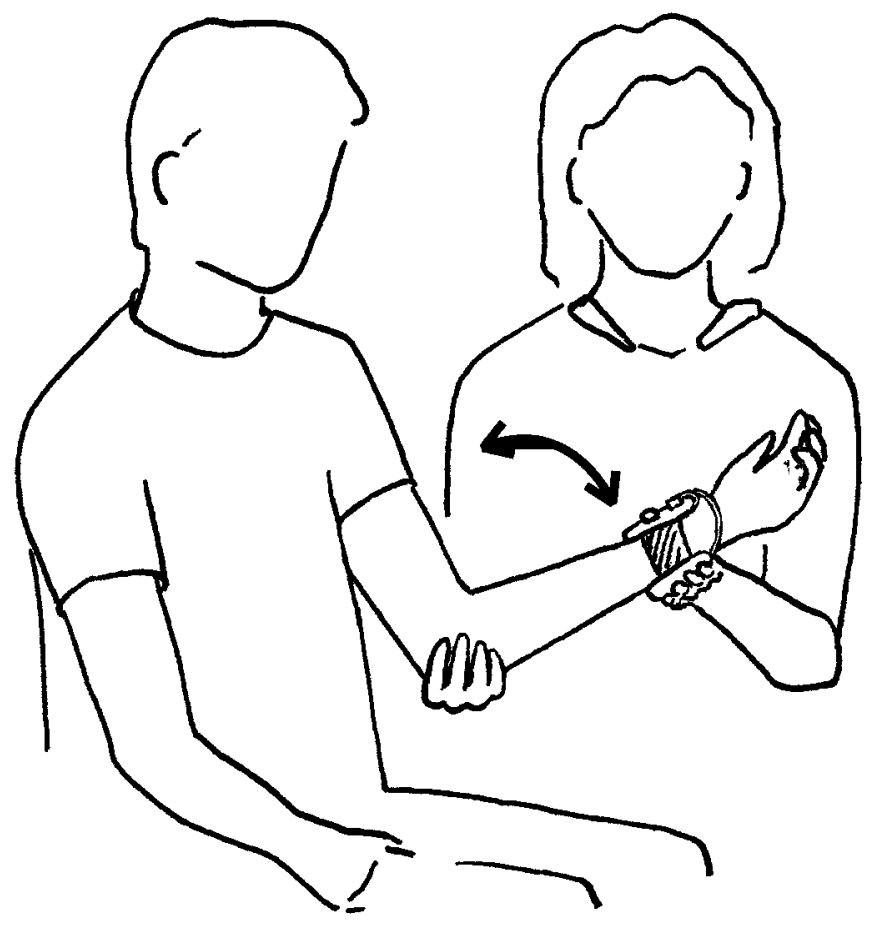

Fig. 1. Use of the rigidity quantification device. To quantify rigidity at the elbow, the examiner flexes and extends the joint through two air-filled pads held around the wrist. The pads are connected to a differential force transducer to monitor the amount of force employed. A gyroscope mounted on one of the pads is used to monitor angular velocity and displacement. A computer program uses these data to calculate elastic stiffness, viscous stiffness, and mechanical impedance. To quantify rigidity at the wrist, the forearm is pronated and the pads held around the hand (not shown).

Calculation of stiffness values is performed by using a leastsquares parametric method to solve the following equation for $K$ and $B$ over a 4-s moving window of data

$$
T=K x+B v+C
$$

where

$T \quad$ torque measured;

$x$ and $v \quad$ angular displacement and angular velocity, respectively;

$K \quad$ elastic stiffness;

$B \quad$ viscosity;

$C \quad$ constant offset of the sensors.

The viscous stiffness is $B \omega$, where $\omega$ equals $2 \pi *$ the mean frequency of the cyclical displacement. The output of the device includes approximately 46-s traces of torque, angular displacement, and event markers, as well as approximately 42-s traces of $K$ and $B \omega$. Mechanical impedance $(Z)$ is the magnitude of the vectorial sum of $K$ and $B \omega$.

Unless otherwise specified, for all procedures described below, raw force and gyroscope signals were taken directly from the output of the data acquisition box $(9.6 \mathrm{~Hz}$ second-order low-pass Sallen-Key filter).

\section{B. Gyroscope Validation}

The performance of the gyroscope was tested against that of a Penny and Giles goniometer (Biometrics, Ltd.) $(\mathrm{P}+\mathrm{G})$, and that 


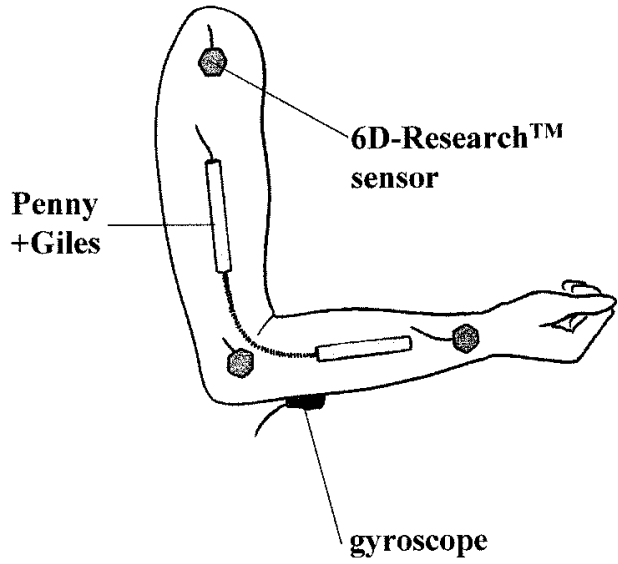

Fig. 2. Positioning of sensors on arm of subject for gyroscope validation. The performance of the gyroscope in monitoring angular displacement of the arm was compared to that of a Penny+Giles goniometer $(\mathrm{P}+\mathrm{G})$, and 3-D movement analysis system (6D-Research). The gyroscope was placed along the ulna, just distal to the elbow, and the $\mathrm{P}+\mathrm{G}$ goniometer was positioned along the elbow on the ulnar side of the arm. Three electromagnetic sensors of the 6D system were placed over the styloid process of the radius, and lateral epicondyle of the humerus, and on the shoulder.

of an electromagnetic three-dimensional (3-D) movement analysis system (6D-Research ${ }^{\mathrm{TM}}$, Skill Technologies, Inc.) (6D).

The sensors were affixed to the right arm of a human subject with no known neurological impairment. The examiner (SP) applied flexion and extension of varying amplitude and frequency to the elbow joint. The subject was asked either to relax or, occasionally, to provide some resistance. Positioning of each of the sensors is shown in Fig. 2. Signals from the gyroscope and $\mathrm{P}+\mathrm{G}$ goniometer were digitized at $20 \mathrm{~s}^{-1}$ [Cambridge Electronic Design (CED) 1401 interface with 12 bit resolution, and SIGAVG (version 5.42) software]. Data from the 6D electromagnetic sensors were sampled at $40 \mathrm{~s}^{-1}$ using the $6 \mathrm{D}$ software. Angular displacement was calculated off-line from the $\mathrm{P}+\mathrm{G}$ goniometer and integrated gyroscope signals using linear interpolation. The $6 \mathrm{D}$ system produced its own calculations of absolute joint angle based on vectorial projections. Calibrated signals were detrended and aligned to reduce any observed phase shift, and the amplitudes of the signals were compared by calculating root mean square (RMS) errors.

\section{Characterization of Force Sensors}

As mentioned above, the force sensors of the device consist of two air-filled pads connected to a differential force transducer. To test linearity, combinations of weights ranging from $10 \mathrm{~g}$ to $4630 \mathrm{~g}$ were applied to one force pad at a time. Data were collected via the Visual Basic user interface and correlation coefficients between the forces applied and the force output signals were computed.

To test the frequency response and phase shifting of the force sensors, sinusoidal variations in force were applied to one of the force pads using a custom-made moving-coil electromagnetic force servo-motor with a 4-strain-gauge proving-ring force transducer. The frequency of the sinusoidal input (Feedback Function Generator FG600) was increased from 0.2 to $10 \mathrm{~Hz}$ over $60 \mathrm{~s}$. The signal from the force sensor and the output of the force transducer of the servo-motor were digitized at 100 $s^{-1}$ (CED 1401 and SIGAVG). A fast Fourier transform was performed in MATLAB on the raw voltage signals; the frequency response and phase shift were determined by dividing the cross spectral density by the power spectrum of the output of the servo-motor force transducer.

\section{Phase Shift Investigation}

Elastic stiffness $(K)$ is the ratio of the component of torque in phase with angular displacement of the joint and the displacement itself; the viscous stiffness $(B \omega)$ is the ratio of the component of torque in phase with angular velocity and the velocity itself. The algorithm used to calculate mechanical impedance first estimates separately the contributions of $K$ and $B \omega$ to the torque measured. The mechanical impedance is then the vectorial sum of the two stiffnesses. Since any phase shifts of the displacement or force signals with respect to each other will affect the relative proportion of $K$ and $B \omega$ calculated by the algorithm, it is important that the sensors do not introduce phase lags or leads.

This was tested for the force sensors as described above. To test the gyroscopes, two gyroscopes were attached to the arm of an angular servo motor (Printed Motors Ltd. "Servalco" servomotor) which moved through a range of $201^{\circ}$ or $91.5^{\circ}$ at frequencies ranging from 0.2 to $1.5 \mathrm{~Hz}$. Signals from the gyroscope and displacement transducers of the servo-motors were digitized at 100 or $20 \mathrm{~s}^{-1}$ (CED 1401 and SIGAVG). The displacement transducer and integrated gyroscope signals were detrended and calibrated by linear interpolation. The calibrated signals were plotted and compared graphically in MATLAB.

\section{E. Validation of System and Test-Retest Reliability on a Model Arm}

In order to validate the results of the stiffness calculations, we quantified the elastic stiffness of a model constructed from a prosthetic arm and compared these measurements to the estimates obtained using the rigidity quantification device. The model is depicted in an inset of Fig. 3(a). Different levels of stiffness were created by attaching different combinations of elastic cords across the elbow. To obtain an independent estimate of stiffness, the arm was flexed and extended via a load cell at about $0.5 \mathrm{~Hz}$ over a constant range measured by a goniometer. Angular displacement was measured using a custom-built linear variable displacement transducer (LVDT). This signal was low-pass filtered at $2.9 \mathrm{~Hz}$ (first order) and $10 \mathrm{~Hz}$ (second order Sallen-Key). LVDT and load cell signals were digitized at $20 \mathrm{~s}^{-1}$ (CED 1401 and SIGAVG). Five 50-s tests were performed at each of four stiffness settings. The stiffness was taken as the slope of the regression line of the plot of angular displacement versus imposed torque.

After each set of five trials of a particular stiffness setting, the stiffness was tested again at the same angular range and frequency for five trials using the rigidity quantification device. The gyroscope was mounted on the ulnar side of the model forearm, just distal to the elbow. For each trial, the mean elastic stiffness $K$ was determined using two methods: 1 ) by the analysis software as described above; and 2) from the slope of the regression line of the angle-torque plot. These two values were then compared to the corresponding estimates of $K$ determined 


\section{Validation of elastic stiffness calculations}

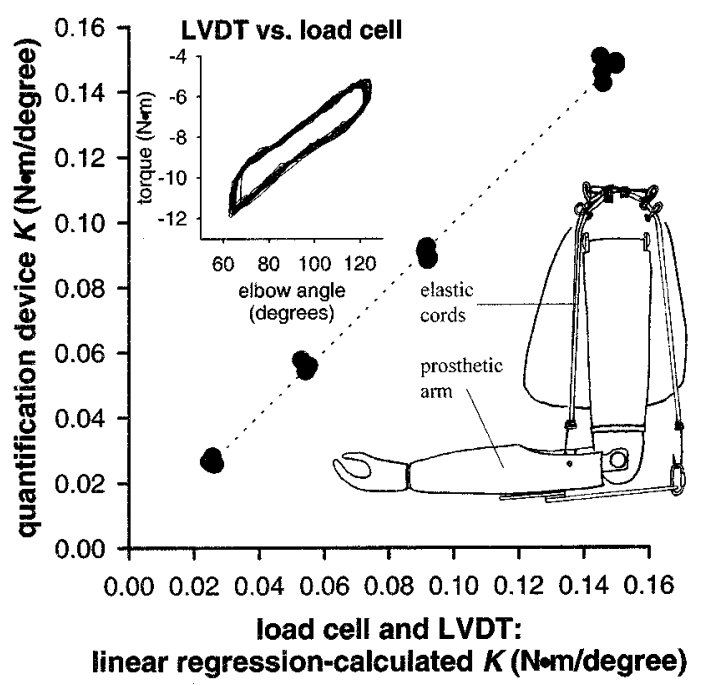

(a)

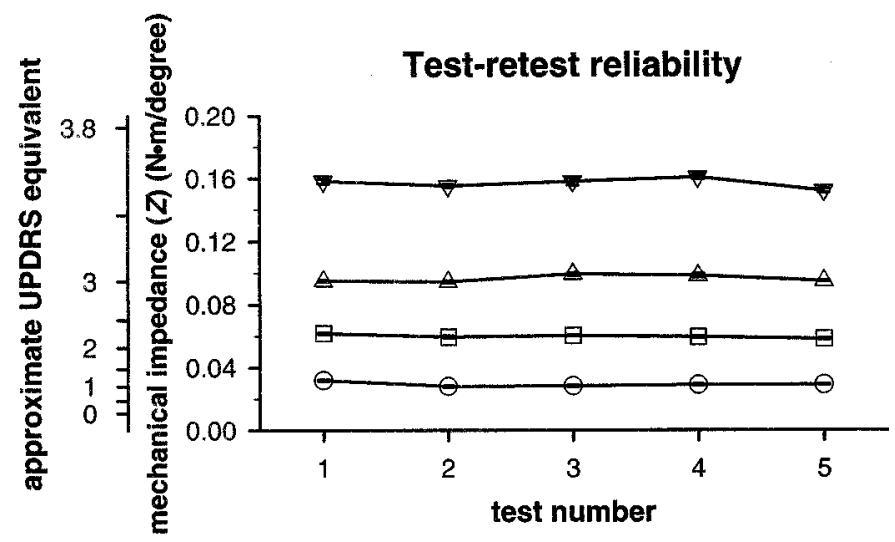

(b)

Fig. 3. Validation of calculations of elastic stiffness using a model arm. The quantification device was tested on a prosthetic limb to which were attached combinations of elastic cords to produce different levels of constant stiffness [schematic inset of (a)]. Stiffness of the system was first estimated by repeatedly flexing and extending the arm over a period of 50 seconds using a load cell and monitoring the angular displacement with an LVDT. The elastic stiffness $(K)$ was taken as the slope of the regression line of the angle-torque plot of the two signals [graph inset of (a)]. Stiffness of the system was subsequently measured using the quantification device. Comparison of the estimates of $K$ calculated by the software of the device to the values determined using the load cell and LVDT yielded a regression coefficient of 0.999 , and absolute values of $K$ were similar (a). These data also offered a check of test-retest reliability of the quantification device on a system of constant stiffness. Shown are the mean of samples of $Z$ $( \pm$ SEM) of individual trials (b). Statistically significant differences between some of the measurements of the same stiffness level are attributed to the small standard errors, and are negligible on the UPDRS scale. The nonlinear equation used to create the UPDRS axis from the $Z$ axis was determined from the clinical data in this paper (see Section III , part E).

from the load cell and LVDT using Pearson's correlation coefficient and linear regression.

These tests also allowed evaluation of the test-retest reliability of the system, as five quantification trials were carried out at each level of stiffness. By taking every $80^{t} h$ data point of the mechanical impedance $Z$ trace (one point every four seconds), 11 independent samples of $Z$ were obtained for each trial. One-way ANOVA with Student-Newman-Keuls test posthoc was used to test for significant differences between the trials for each stiffness setting. When the data sets failed tests of normality or equal variance, nonparametric versions of the statistical tests were used (Kruskal-Wallis ANOVA and Student-Newman-Keuls test). Alpha was set at 0.01 for ANOVA, and 0.05 for the posthoc tests.

\section{F. Clinical Testing and Determination of Relationship of Mechanical Impedance to Qualitative UPDRS Ratings}

Clinical testing of the device was performed in order to investigate the intra- and inter-rater reliability of measures of $Z$ produced by the device, and to compare this reliability to that of the rigidity scale of the UPDRS. In addition, we wished to compare the relationship between $Z$ and the clinical ratings for rigidity at the elbow to that determined using an older version of the device [9], as well as to describe such a relationship for rigidity at the wrist.

Four patients with idiopathic Parkinson's disease consented to participate in this study in accordance with local ethical committee approval. The average age of the subjects was 58 years (range 52 to 64 years), and the average duration since onset of symptoms was 8 years (range 6 to 10 years). One subject (GM) had undergone a right unilateral pallidotomy two years prior to this study. All subjects were tested without alteration of their daily medication routine; thus, at the time of testing, subjects were not necessarily receiving maximal pharmacological benefit.

Four examiners also participated in this study. JJ and WM are neurologists, and MW is a physiotherapist; all three had extensive experience in clinical evaluation of parkinsonian rigidity. AP, one of the authors of this paper, and one of the developers of the quantification device, was less experienced in the clinical evaluation of rigidity at the elbow. JJ and AP had both participated in a previous study involving the quantification device [9].

The rigidity of each patient was evaluated by each examiner. The wrist and elbow of the right arm were assessed, separately, in all cases. The examiner first rated the rigidity of the joints using his or her regular method. Then one or two quantification trials lasting $50 \mathrm{~s}$ each were performed on each joint. The examiners instructed the patient to perform a reinforcement manoeuvre of the examiner's choice (e.g., clench contralateral fist, or tap leg with contralateral hand) for roughly half of each trial. For the quantification trials, the forearm was supinated while the elbow was tested, and pronated while the wrist was tested. Every few seconds over the course of each trial, as well as when the rigidity level was felt to change, the examiner verbally rated the rigidity according to the UPDRS; this rating was recorded by SP using the keypad. For all subjects except one, the order of examiners was: JJ, WM, MW, AP; for subject WD, the order of $\mathrm{JJ}$ and $\mathrm{WM}$ was switched.

For purposes of statistical analysis, we assumed that independent samples of $Z$ could be obtained from a single 50-s trial by taking samples every four seconds (every $80^{t} h$ point). (Since negative $K$ or $B \omega$ values imply that the subject was assisting the movements imposed by the examiner, data points falling within a segment of the trace where either $K$ or $B \omega$ was negative were excluded; the point immediately after such a segment was selected instead.) The corresponding data points from the keypad trace were used as independent samples of the clinical rating. 
The samples were grouped according to whether or not they were collected during periods of reinforcement. Any samples from nonreinforced segments occurring after a reinforced segment in the same trial were omitted, as effects of reinforcement may still have been present.

To determine the relationship between $Z$ and the clinical rating (UPDRS), the average of the samples of nonreinforced or reinforced $Z$ obtained from one trial was plotted against the average of the corresponding samples of clinical rating. The linear relationship was described as the slope of the regression line passing through the origin and fitted to data from all trials performed during the study. A nonlinear relationship was also determined by fitting an exponential equation to the data (SigmaPlot ${ }^{\circledR}$ Version 4.01, Jandel Scientific).

Usually an examiner performed two trials of impedance quantification on each subject. Scores of nonreinforced or reinforced rigidity ( $Z$ or clinical rating) of one subject by one examiner could be calculated by taking the average of all such samples obtained from both trials. A combined rigidity score ( $Z$ or clinical rating) was obtained by taking the average of the nonreinforced and reinforced scores; this allowed equal weighting of either score so that the final measurement was not contingent on the amount of time allotted to reinforcement maneuvers.

The Spearman rank-order correlation coefficient $\left(r_{s}\right)$ was used as a measure of test-retest reliability, by calculating $r_{s}$ between the results of the first and second trials performed on the same subject by the same examiner. Agreement between examiners was estimated by comparing the coefficient of variation (standard deviation/mean) for each method of measurement.

Impedance of five normal control subjects was measured by SP using the quantification device. Subjects ranged in age from 46 to 76 years (mean 62.2 years) and included three males and two females. Both wrists were tested in four subjects, and both elbows in all five. Three 50-s tests were performed on each joint. The subjects were asked to perform reinforcement manoeuvres (tap the knee with the other hand) for roughly the last half of the second and third trials. Impedance traces were subsampled as described above. Scores of nonreinforced and reinforced $Z$ were calculated for each subject using the data from all trials for one joint combined.

Unless otherwise stated, statistical analysis for all of the above methods was performed using SigmaStat ${ }^{\mathrm{TM}}$ Version 1.0 (Jandel Scientific).

\section{RESULTS}

\section{A. Gyroscope Validation}

Passive angular displacement of the elbow joint of one subject was measured using a gyroscope, $\mathrm{P}+\mathrm{G}$ goniometer and threedimensional movement analysis system (6D). The RMS error of the gyroscope compared to $\mathrm{P}+\mathrm{G}$ goniometer (mean $\pm \mathrm{SEM}$ $\left.2.1 \pm 0.09^{\circ}\right)$ or $6 \mathrm{D}$ system $\left(1.9 \pm 0.12^{\circ}\right)$ represented less than $3 \%$ of the total range tested.

\section{B. Characterization of Force Sensors}

The linearity of the force sensors was tested by applying weights of increasing magnitude to each of the force pads.
Correlation between the weight and the force output signal was excellent, regression coefficients $(r)$ ranging from 0.997 to 1.000 for the trials using weight combinations ranging from 500 to $3630 \mathrm{~g}$. Deviation from the regression line was $3 \%$ over \pm 3630 g. Regression coefficients were equally good when smaller weights were applied ( 10 to $500 \mathrm{~g}, r=0.998$ to 1.000 ), as well as when larger weights were investigated (1000 to $4630 \mathrm{~g}, r=0.988$ to 0.999 ). The range of weights applied corresponds to the forces used to passively move wrist and elbow joints of control subjects or subjects with mild to severe parkinsonian rigidity.

The frequency response and phase shift of the force sensors was tested by applying sinusoidal variations in force to one of the force pads via a linear servo-motor in force feedback mode. Compared to the output of the proving- ring force transducer of the servo-motor, the change in gain of the pad force sensor was $2 \mathrm{~dB}$ in the range of 0.2 to $10 \mathrm{~Hz}$, and $0.5 \mathrm{~dB}$ over 0.2 to 2.0 $\mathrm{Hz}$. The force pad signal lagged that of the force transducer of the servo-motor strain gauge by $0.5^{\circ}$ at $0.5 \mathrm{~Hz}$, and $2^{\circ}$ at $1 \mathrm{~Hz}$. This was opposite to the lead expected based on the frequency response, and is thought to be caused by a delay due to the time taken for the air to move through the tube connecting the force pad to the differential force transducer. We conclude that the force pad transducers are linear and have a flat frequency response over the range of frequencies of movements that would be imposed during rigidity evaluation.

\section{Phase Shift Investigation}

Potential phase shift of the integrated gyroscope signal with respect to the output of length transducers of servo-motors was investigated.

When the gyroscope was tested over a $90^{\circ}$ or $200^{\circ}$ range and its signal sampled at $20 \mathrm{~s}^{-1}$, the integrated signal (angular displacement) generally lagged the output of the length transducer of the servo-motor by 20 to $30 \mathrm{~ms}$ (equivalent to about $4.5^{\circ}$ phase lag at $0.5 \mathrm{~Hz}$, and $9^{\circ}$ phase lag at $1 \mathrm{~Hz}$ testing frequencies). The phase shift was negligible when the gyroscope signal was sampled at $100 \mathrm{~s}^{-1}$, and this suggests that the observed phase shift at $20 \mathrm{~s}^{-1}$ was a result of integration at a low frequency of sampling and not an artifact of the sensor itself.

During evaluation of rigidity, the joint is generally tested over an approximately $90^{\circ}$ range, at a rate of 0.5 to $1 \mathrm{~Hz}$. When these ranges are considered, our results indicate that the relative phase shift between the force and angular displacement signals is small $\left(4^{\circ}\right.$ to $\left.7^{\circ}\right)$, and the effect on estimates of $K$ and $B \omega$ minimal.

\section{Validation of System and Test-Retest Reliability on a Model Arm}

The stiffness calculations of the quantification device were validated by comparing device-generated estimates of $K$ to the stiffness of a model arm measured in separate tests with other transducers [Fig. 3(a)]. The four stiffness settings tested covered the general range of elbow stiffnesses we have encountered in patients with parkinsonian rigidity. There were excellent correlations between the independent estimates of $K$ obtained with the LVDT and load cell and those computed by the device software $[r=0.999$, Fig. 3(a)], or determined from angle-torque 
plots generated from the output of the device $(r=0.998$, not shown). Absolute values were very similar; the slopes of these regression lines were 0.983 and 0.954 , respectively.

Since eleven independent samples of $Z$ could be obtained from each trial that employed the quantification device, this set of experiments presented an opportunity to evaluate the test-retest reliability of the device at each of four relatively constant stiffness levels. Individual trials within a stiffness setting were usually statistically different from each other (ANOVA or Kruskal-Wallis ANOVA, $\alpha=0.01$, and Student-Newman-Keuls test posthoc, $\alpha=0.05$ ). However, the standard error of each test was very small; although the values were different statistically, they were not different in a practical sense given that the variability corresponded to a small fraction of one point on the UPDRS scale [see Fig. 3(b)].

\section{E. Clinical Testing and Relationship Between $Z$ and UPDRS}

Four examiners measured the mechanical impedance of the right elbow and wrist of four patients with idiopathic Parkinson's disease using the quantification device. They also rated the rigidity of the joints on the UPDRS scale both during the quantification trials $\left(\mathrm{UPDRS}_{\text {during }}\right.$ ) as well as separately using their usual method before the trials $\left(\mathrm{UPDRS}_{\text {usual }}\right)$. Reinforcement manoeuvres varied between examiners and subjects and were chosen by the examiner. When the quantification device was employed, the period during which the subject performed reinforcement maneuvers ranged from one third of the trial to the entire test period, as decided by the examiner. One of the subjects (GM) experienced medication-induced dyskinesias throughout the session.

Without reinforcement, $Z$ ranged from 0.00803 to 0.1058 $\mathrm{N} \bullet \mathrm{m} /$ degree (mean \pm SEM: $0.0419 \pm 0.0219)$ for the elbow and 0.00345 to $0.0254 \mathrm{~N} \bullet \mathrm{m} /$ degree (mean \pm SEM: $0.0114 \pm$ 0.00498 ) for the wrist. Reinforcement produced overall statistically significant increases in $Z$ (paired $t$-test, $p<0.05$ ) for both joints ( $Z$ with reinforcement: elbow: range 0.0178 to $0.1433 \mathrm{~N} \bullet \mathrm{m} /$ degree, mean \pm SEM: $0.0638 \pm 0.0279$, wrist: range 0.00890 to $0.0363 \mathrm{~N} \bullet \mathrm{m} /$ degree, mean \pm SEM: 0.0194 $\pm 0.00652)$

When quantified measures $(Z)$ of reinforced and nonreinforced rigidity from each trial performed at the elbow were plotted against simultaneously-obtained UPDRS $_{\text {during }}$ scores, the slope of the regression line fitting the data from all trials was 27.4, giving a rough description of the relationship between $Z$ scores and the UPDRS rigidity scale. However, it is evident from the plot that $Z$ and clinical ratings are not linearly related (Fig. 4). In attempt to describe this relation more accurately, the data were fitted with an exponential equation in the form

$$
\text { UPDRS rating }=a\left(1-e^{b(c-Z)}\right) \text {. }
$$

To take into account a nonzero $Z$ for control subjects, $c$ was set to 0.0108 (mean $Z$ of control subject elbow data, without reinforcement). Further, the parameter $a$ was set to 4 to reflect the maximum possible UPDRS rating. The result was the following equation:

$$
\text { UPDRS rating }_{\text {elbow }}=4\left(1-e^{16.5(0.01-Z)}\right) .
$$

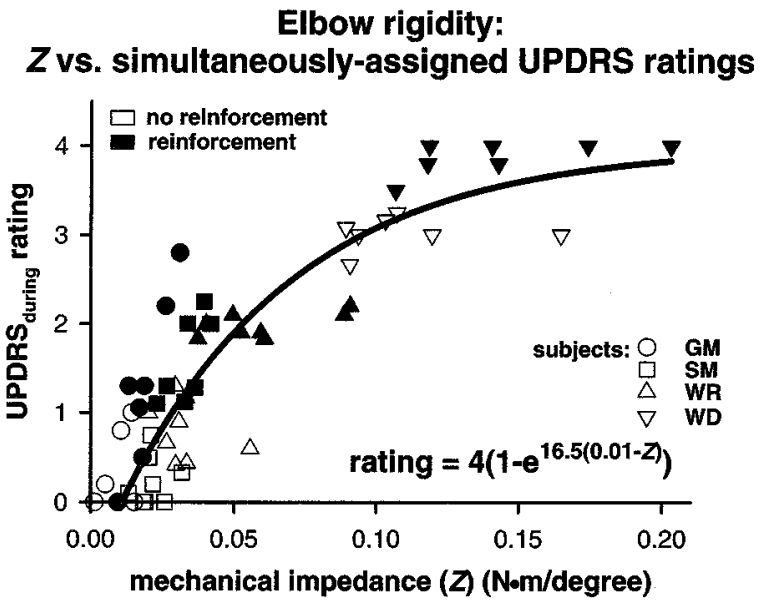

Fig. 4. Relationship between quantified measures and clinical ratings of elbow rigidity. Quantified measures of mechanical impedance $(Z)$ were plotted against simultaneously-assigned UPDRS ratings obtained during quantification trials $\left(\mathrm{UPDRS}_{\text {curing }}\right)$ showing a nonlinear relationship. The data were fitted by the line in the figure; the equation of this line was used as a means to express $Z$ in terms of the UPDRS rigidity scale. Each point represents the mean of samples of nonreinforced (open symbols) or reinforced (filled symbols) rigidity from one trial.

Using this equation, $Z$ can be scaled so that $Z$ and UPDRS scores for individual subjects can be compared graphically. Fig. 5(a) demonstrates the correlation between $Z$ and UPDRS $_{\text {during }}$ scores shown in Fig. 4, and also indicates the variation in scores for each subject. Each large point represents the mean \pm standard deviation of four scores (one mean score from each examiner). The smaller circles represent the mean scores of individual examiners for each subject. The combined rigidity score of an examiner for a subject is the average of the nonreinforced and reinforced rigidity scores; these are plotted in Fig. 5(b). Combined scores are compared to the UPDRS ${ }_{\text {usual }}$ ratings, for which a single score takes both reinforced and nonreinforced rigidity into account.

In general, interexaminer agreement for rigidity at the elbow was greater with higher rigidity levels. Across subjects, the mean $( \pm$ SEM) coefficients of variation for raw $Z$ values and UPDRS $_{\text {during }}$ scores were $0.35 \pm 0.17$ (range 0.16-0.84) and $0.59 \pm 0.22$ (range 0.03-0.99), respectively, for nonreinforced rigidity and $0.31 \pm 0.06$ (range $0.20-0.44$ ) and $0.33 \pm 0.20$ (range 0.04-0.900), respectively, for reinforced rigidity. Taking both nonreinforced and reinforced rigidity into consideration, mean \pm SEM coefficients of variation across subjects were $0.17 \pm 0.06$ (range 0-0.29) for UPDRS usual $_{\text {scores, }} 0.32 \pm$ 0.16 (range $0.03-0.74$ ) for combined UPDRS during $_{\text {s }}$ scores, and $0.28 \pm 0.08$ (range $0.14-0.50$ ) for raw $Z$ values. Coefficients of variation were most consistent between subjects for UPDRS $_{\text {usual }}$ scores.

For combined rigidity scores, the maximum difference in UPDRS $_{\text {usual }}$ for any subject was 1.5 , comparable to the maximum difference between UPDRS $S_{\text {during }}$ of 1.4. Combined $Z$ values scaled using (3) demonstrated a maximum intrasubject difference of 1.0. The average maximum difference in scores across subjects was comparable for all three measures $(0.63-0.73)$. 


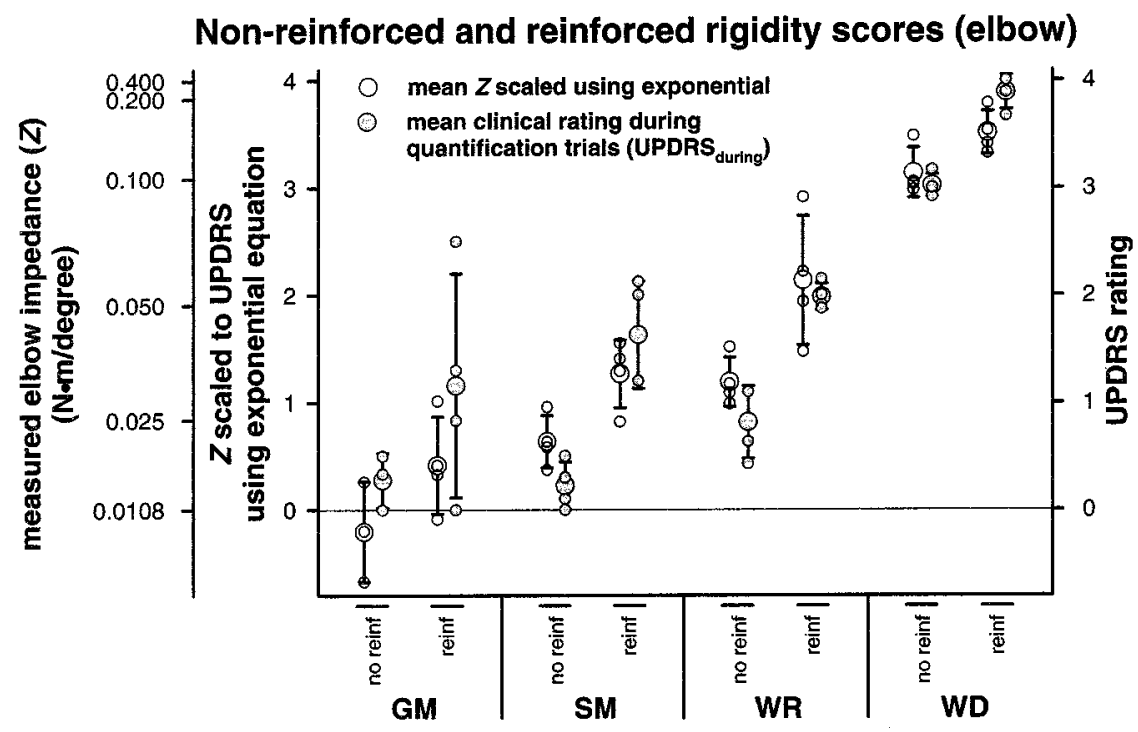

(a)

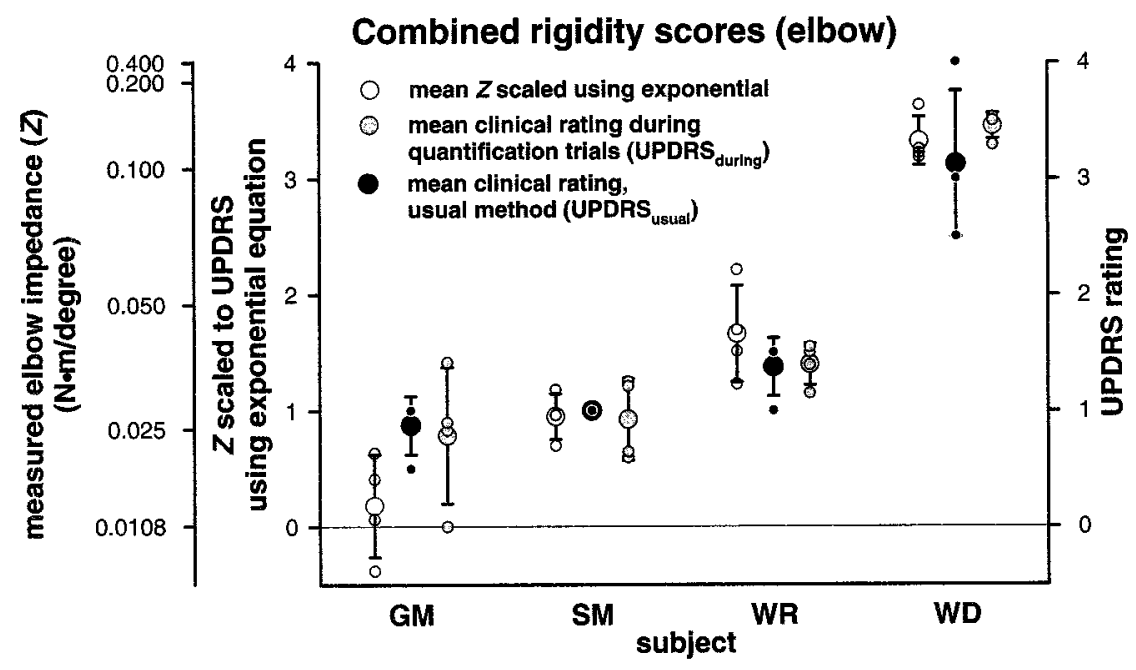

(b)

Fig. 5. Correlation between and interexaminer agreement of scaled $Z$ and UPDRS scores of elbow rigidity. Raw $Z$ measures were converted to the UPDRS scale using the exponential in Equation (3) and compared to UPDRS ratings. Each large data point represents the mean \pm standard deviation of four scores of rigidity (one from each examiner); the size of the error bars reflects the level of interrater agreement. Small data points represent the mean score from individual examiners

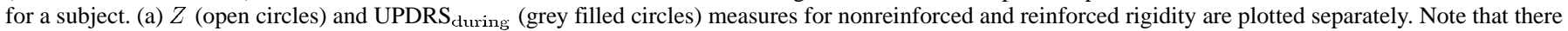
are no measures of nonreinforced rigidity for subject GM from examiner JJ. (b) UPDRS $_{\text {usual }}$ scores (black filled circles) take into account both reinforced and

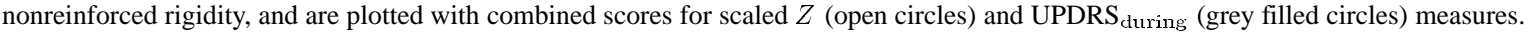

Test-retest reliability at the elbow, as indicated by Spearman's rank order correlation coefficients $\left(r_{s}\right)$ was greatest for combined rigidity scores $\left[r_{s}=0.95\right.$ (raw $Z$ ) or 0.94 (UPDRS during $)$, when nonreinforced and reinforced rigidity scores were pooled $\left(r_{s}=0.91\right.$ or 0.94$)$, or when reinforced rigidity scores were considered separately $\left(r_{s}=0.96\right.$ or 0.93$)$. Reliability was less for measures of nonreinforced rigidity $\left(r_{s}=0.78\right.$ or 0.71$)$. Test-retest reliability could not be estimated for the usual clinical method $\left(\mathrm{UPDRS}_{\mathrm{usual}}\right)$ as each examiner rated the rigidity of a single joint only once.

It is proposed that the $Z$ score may offer a more accurate description of changes of rigidity with reinforcement. In Fig. 4, most of the points of reinforced rigidity (black points) lie above the fitted line, suggesting a tendency of the examiners to over-rate rigidity on the UPDRS scale when they know that reinforcement manoeuvres are being performed.

In general, the observations from the measurements of rigidity at the elbow hold for those at the wrist, although the picture is not as clear with the wrist data. The plot of raw $Z$ values versus UPDRS $_{\text {during }}$ ratings yielded a linear regression line with a slope of 76.2. As with the elbow, the relation between $Z$ and UPDRS $_{\text {during }}$ at the wrist is nonlinear and the equation

$$
\text { UPDRS rating }_{\text {wrist }}=4\left(1-e^{37(0.001-Z)}\right)
$$

was fitted to the data (Fig. 6).

Interexaminer agreement for clinical ratings (UPDRS during $_{\text {}}$ and UPDRS $_{\text {usual }}$ ) was generally better at higher levels of wrist 


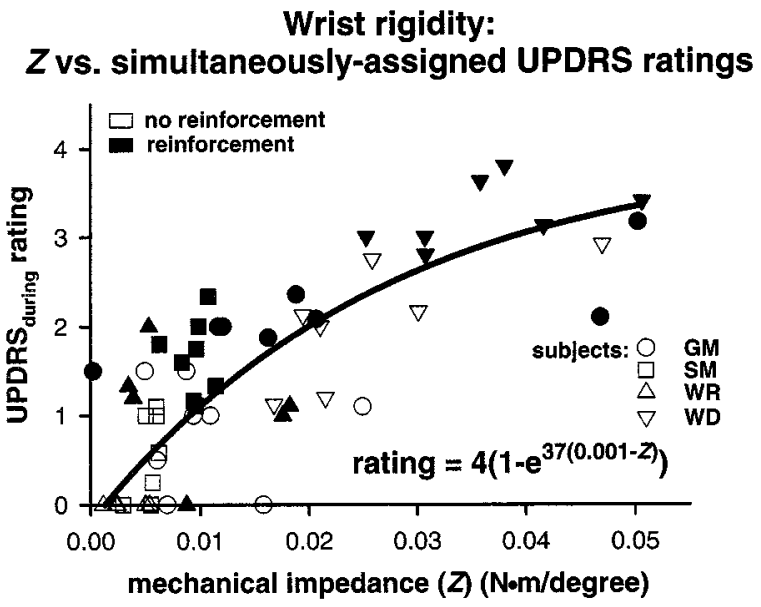

Fig. 6. Relationship between quantified measures and clinical ratings of wrist rigidity As with the elbow, an exponential equation (inset) was fitted to the plot of raw $Z$ values versus simultaneously-obtained UPDRS $_{\text {during }}$ scores of rigidity at the wrist. Each symbol represents the mean of samples of nonreinforced or reinforced rigidity from one trial.

rigidity, whereas agreement between raw $Z$ values appeared to be independent of level of impedance. Coefficients of variation of raw $Z$ values and $\mathrm{UPDRS}_{\text {during }}$ scores ranged from 0.19 to 0.65 (mean \pm SEM $0.45 \pm 0.10$ ) and from 0.37 to $0.80(0.57$ \pm 0.13 ), respectively, for nonreinforced rigidity, from 0.17 to $0.73(0.45 \pm 0.16)$ and from 0.08 to $0.77(0.30 \pm 0.16)$, respectively, for reinforced rigidity, and from 0.13 to $0.69(0.43$ $\pm 0.14)$ and from 0.11 to $0.77(0.33 \pm 0.15)$, respectively, for combined scores. Coefficients of variation for $\mathrm{UPDRS}_{\text {usual }}$ ratings ranged from 0.21 to $0.67(0.39 \pm 0.10)$. Large variation in $Z$ during reinforcement for subject WR is attributed to two things. First, examiner AP instructed a stronger reinforcement manoeuvre than had the other examiners. Second, WR assisted the limb movements imposed by examiner MW for most of the two trials, the result being that only a few data samples were obtained from each trial. With the exception of subject WR, agreement of raw $Z$ scores was comparable to or better than agreement between $\mathrm{UPDRS}_{\text {usual }}$ ratings.

For combined wrist rigidity measures, the maximum difference in scores for a subject was comparable between UPDRS $_{\text {usual }}(1.5)$ and $Z$ [scaled using (4), 1.7], and lowest for $\mathrm{UPDRS}_{\text {during }}(1.0)$. The average maximum difference across

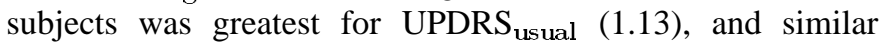
between $\mathrm{UPDRS}_{\text {during }}(0.76)$ and $Z$ (scaled, 0.92)

Spearman rank order correlation coefficients between scores obtained from first and second trials at the wrist, reflecting test-retest reliability, were better for $Z$ values $\left(r_{s}=0.88\right.$ to 0.91$)$ than for UPDRS $_{\text {during }}$ scores $\left(r_{s}=0.80\right.$ to 0.89$)$. For quantified measures, coefficients were equally good for nonreinforced as for reinforced and combined rigidity scores; coefficients for $\mathrm{UPDRS}_{\text {during }}$ ratings were slightly lower with nonreinforced rigidity.

Nonreinforced $Z$ values for control subjects ranged from 0.0045 to $0.0216 \mathrm{~N} \bullet \mathrm{m} /$ degree (mean \pm SEM: $0.0108 \pm 0.0024$ ) for the elbow and 0.00040 to $0.00264 \mathrm{~N} \bullet \mathrm{m} /$ degree $(0.00129 \pm$ 0.00028 ) for the wrist. Often subjects had difficulty in relaxing the arm, resulting sometimes in resistance to the passive movements imposed. Reinforcement had no effect on impedance at the wrist in control subjects. At the elbow, impedance was seen to increase by as much as $273 \%$ with reinforcement, but this was inconsistent; across subjects, reinforced impedance was slightly higher but statistically insignificant than nonreinforced impedance. Note that because negative values of $K$ or $B \omega$ resulted in exclusion of data, the values presented above represent the results from seven elbows (four subjects) and seven wrists (four subjects). Data of reinforced impedance at the wrist was only available from six joints (three subjects).

\section{DISCUSSION}

The data presented here demonstrate that a device introduced previously [9] provides a valid and effective means of quantifying rigidity of the upper limb.

The gyroscope, used to monitor angular displacement of the limb, is accurate within 5\%. The force sensors are linear with a frequency response that is flat over the maximum range of frequencies of passive limb movement anticipated under the conditions of clinical evaluation of rigidity.

Results obtained from the quantification device were validated with those of another measure of stiffness on a model arm. Trials performed using this model arm as well as clinical trials on subjects with Parkinson's disease demonstrated good test-retest reliability of quantified measures.

In clinical trials, $Z$ measures corresponded well to clinical ratings of parkinsonian rigidity, both in individual trials and across the data set as a whole. The slope of the regression line fitting $Z$ and UPDRS $_{\text {during }}$ data from the elbow (27.4) was close to that obtained in our previous study (22.9) [9]. Two examiners (AP, JJ) had participated in the earlier trials, but all subjects were unique to the present study. In the current study, we have determined that the relationship between $Z$ and clinical ratings is best described with a nonlinear equation. This is in agreement with the observations of Weber, Fechner, and Stevens that, in general, perception is nonlinearly related to stimulus magnitude [26]. While Fechner expressed the sensation-stimulus relationship in terms of a logarithmic function, Stevens later determined that the relationship was better described by a power law, which could be applied to noxious as well as neutral stimuli [26]. We chose to use an exponential equation in order to accommodate for the upper limit imposed on the UPDRS scale.

In our previous study, it was suggested that the slope of the regression line be used as a conversion factor with which to express $Z$ measurements in terms of UPDRS ratings. Though a linear conversion factor is simpler to understand and to apply, the exponential equation offers a more accurate picture of the relation between $Z$ and the UPDRS rigidity scale. There exists a nonlinear relationship between stiffness and its perception: what feels twice as stiff actually is not. The exponential equation expresses the perception of mechanical impedance better than a linear conversion.

Do (3) and (4) hold for all subjects? In our experience, the answer is a qualified yes, though in very slight subjects the clinical rating may be somewhat underestimated by the equations (clinicians presumably take into account some estimate of a subject's 
potential strength). Taking into consideration that the analysis procedure of the rigidity quantification device does not incorporate a correction for limb mass, and that linearity of measurement has been listed as an advantage of quantification systems [21] (a joint twice as stiff produces a measure with twice the magnitude), we suggest that rigidity measurements should eventually be reported in terms of Système International (SI) units rather than UPDRS ratings. In the mean time, the exponential equations (3) and (4) provide a means whereby one may interpret, in general, the meaning of such measures with respect to the clinically intuitive UPDRS scale.

It was observed that measurement of mechanical impedance of the elbow of a completely relaxed control subject without hypertonus tends to produce negative values of $K$. This is likely due to the varying action of gravity on the mass of the forearm as it is rotated. Our current method of discarding any segment of $Z$ containing negative values of either $K$ or $B \omega$ does not accommodate for situations of low stiffness with complete relaxation. Testing our model arm without elastic cords (i.e., no stiffness; the weight of this arm was comparable to that of a human arm) produced negative values of $K$ ranging from -0.0018 to $-0.0083 \mathrm{~N} \bullet \mathrm{m} /$ degree $($ mean \pm SEM: $-0.0045 \pm 0.00005$ ). This needs to be tested on human subjects showing no electromyographic activity of the muscles of the upper arm.

In the current study, interrater agreement and test-retest reliability of quantified measures of impedance were similar to those of UPDRS ratings of rigidity. The conclusion may then be drawn that these measures are redundant and that clinical evaluation alone would suffice. However, it is important to keep in mind that the examiners involved in this study had worked together previously, and three of them hadbeeninvolved in the firstclinical assessments of the quantification device. Some studies have reported moderate to excellent interrater reliability of the rigidity component of the UPDRS scale, but in at least two of these studies comparisons were made between examiners from the same center [4], [5], [27] . Another study evaluating the Columbia University and Webster scales involved four examiners from different centers [8]. The relatively poor reliability reported was attributed not only to the inexperience of the raters, but also to differences in technique employed and interpretation of the scales. Another study evaluating these same two scales with six neurologists from the same center reported only "fair" to "moderate" interrater reliability [7], perhaps an indication of shortcomings in the scales themselves. In any case, differences in the method of rigidity assessment is one source of disagreement between clinicians.

In an unpublished clinical trial of our quantification device at the National Hospital, Queen Square, London, U.K., five neurologists from three countries assessed rigidity at the elbow and wrist of seven subjects with Parkinson's disease. Their UPDRS ratings for a given patient differed by as much as two points. However, their ratings subsequently collected over 50-second trials with the device (UPDRS during $_{\text {) }}$ tended to show better agreement. The improvement was apparently due to specification of the plane of movement of the joint and prolongation of the length of the test. The need for uniformity in methods of application of rating scales has long been recognized [1], [28], [29]. It has been recommended that clinicians involved in a study establish agreement on the technique to be used and interpretation of scales [8], [30]. However, this does not remedy the problem of comparing results between studies.

Quantification of rigidity can help by removing the subjective element of the scales. In the London study above, persisting interrater discrepancies between $\mathrm{UPDRS}_{\text {during }}$ scores obtained under the more controlled conditions of quantification of rigidity were attributed largely to individual interpretation of the scale. In the present study, it is thought that the quantified measures more accurately reflected increases in rigidity with reinforcement maneuvers.

It is important to note that standardization of technique is also desirable when a quantification device is employed, since many variables can affect the level of muscle tone observed [1]. In the present study, occasional large variability in measures of mechanical impedance was most likely due to actual differences in the level of rigidity evoked. For example, examiner AP instructed subject WR to perform a reinforcement manoeuvre that was stronger than requested by the other examiners.

In this paper, we have demonstrated validity and reliability of a device for the quantification of parkinsonian rigidity. The device fulfills the requirements demanded by a clinical setting, that is, it is relatively inexpensive, time-efficient, and simple. The way in which this device is used more closely emulates the clinical examination than other methods of quantitative assessment. Measurements are sensitive to changes in impedance levels due to reinforcement, as well as to fluctuations in rigidity which occur spontaneously during the course of a trial and which are detectable by the clinician. The nonlinear relationships between $Z$ and simultaneously-acquired UPDRS scores of rigidity at the elbow and wrist were described using exponential equations. Because differences in technique of rigidity assessment and interpretation of rating scales appear most evident when clinicians from different centers are compared, a study involving a large, international sample of clinicians would be desirable to validate and possibly refine these equations.

The quantification device evaluated here is currently in use in several clinical and research settings in North America and Europe and the number is slowly growing. If the device achieves widespread acceptance, then the choice will need to be made as to whether to communicate the measures of rigidity in terms of mechanical impedance or as scores relating to the UPDRS scale. Scientifically, the former approach is preferable, but it may be that the method will gain better initial acceptance if measures are interpreted in terms of the familiar clinical rating scales. Expression of $Z$ in terms of UPDRS ratings using equations such as those determined here may subsequently allow a gradual transition to the expression of rigidity in the standard physical units of the Système International (SI).

\section{ACKNOWLEDGMENT}

The authors would like to thank J. H. Jhamandas, W. R. Martin, and M. Wieler for their valuable participation in this study. They would also like to express appreciation for the involvement of $\mathrm{J}$. C. Rothwell, P. Limousin, F. Yokoshi, C. D. Marsden, N. Quinn, and P. Brown in an earlier clinical trial of the quantification device at the Institute of Neurology, National Hospital, Queen Square, 
London, U.K. In addition, they would like to thank D. J. Bennett and K. Yoshida for their expertise and help with data analysis.

\section{REFERENCES}

[1] A. E. T. Lang and S. Fahn, "Assessment of Parkinson's disease," in Quantification of Neurological Deficit, T. L. Munsat, Ed. Boston, MA: Butterworths, 1989, pp. 285-309.

[2] P. Martínez-Martín, "Rating scales in Parkinson's disease," in Parkinson's Disease and Movement Disorders, 2nd ed, J. Jankovic and E. Tolosa, Eds. Baltimore, MD: Williams and Wilkins, 1993, pp. 281-292.

[3] C. D. Marsden and M. Schachter, "Assessment of extrapyramidal disorders," Br. J. Clin. Pharmacol., vol. 11, no. 2, pp. 129-151, 1981.

[4] J. M. Rabey, H. Bass, U. Bonuccelli, D. Brooks, P. Klotz, A. D. Korczyn, P. Kraus, P. Martínez-Martín, P. Morrish, W. Van Sauten, and B. Van Hilten, "Evaluation of the short Parkinson's evaluation scale: A new friendly scale for the evaluation of Parkinson's disease in clinical drug trials," Clin. Neuropharmacol., vol. 20, no. 4, pp. 322-337, 1997.

[5] M. Richards, K. Marder, L. Cote, and R. Mayeux, "Interrater reliability of the unified Parkinson's disease rating scale motor examination," Mov. Disord., vol. 9, no. 1, pp. 89-91, 1994.

[6] L. R. Van Dillen and K. E. Roach, "Interrater reliability of a clinical scale of rigidity," Phys. Ther., vol. 68, no. 11, pp. 1670-1681, 1988

[7] A. Ginanneschi, F. Degl'Innocenti, S. Magnolfi, M. T. Maurello, I. Catarzi, P. Marini, and L. Amaducci, "Evaluation of Parkinson's disease: Reliability of three rating scales," Neuroepidemiology, vol. 7, no. 1 , pp. 38-41, 1988.

[8] G. Geminiani, B. M. Cesana, F. Tamma, P. Contri, C. Pacchetti, F. Carella, R. Piolti, E. Martignoni, P. Giovannini, F. Girotti, and T. Caraceni, "Interobserver reliability between neurologists in training of Parkinson's disease rating scales: A multicenter study," Mov. Disord., vol. 6, no. 4, pp. 330-355, 1991.

[9] A. Prochazka, D. J. Bennett, M. J. Stephens, S. K. Patrick, R. SearsDuru, T. Roberts, and J. H. Jhamandas, "Measurement of rigidity in Parkinson's disease," Mov. Disord., vol. 12, no. 1, pp. 24-32, 1997.

[10] C. D. Ward, J. N. Sanes, J. M. Dambrosia, and D. B. Calne, "Methods for evaluating treatment in Parkinson's disease," Adv. Neurol., vol. 37, pp. 1-7, 1983.

[11] H. Teräväinen, J. K. Tsui, and D. B. Calne, "Evaluation of Parkinson's disease," in Drugs for the Treatment of Parkinson's Disease, D. B. Calne, Ed. Berlin, Germany: Springer-Verlag, 1989, vol. 88, Handbook of Experimental Pharmacology, pp. 271-279.

[12] W. G. Ondo, J. Jankovic, E. C. Lai, C. Sankhla, M. Khan, L. Ben-Arie, K. Schwartz, R. G. Grossman, and J. K. Krauss, "Assessment of motor function after stereotactic pallidotomy," Neurology, vol. 50, no. 1, pp. 266-270, 1998 .

[13] J. A. Obeso, G. Linazasoro, J. C. Rothwell, M. Jahanshahi, and R. Brown, "Assessing the effects of pallidotomy in Parkinson's disease [letter]," Lancet, vol. 347, no. 9013, p. 1490, 1996.

[14] D. D. Webster, "A method of measuring the dynamic characteristics of muscle rigidity, strength, and tremor in the upper extremity," IRE Trans. Med. Electron., pp. 159-164, Sept. 1959.

[15] H. Teräväinen, J. K. C. Tsui, E. Mak, and D. B. Calne, "Optimal indices for testing parkinsonian rigidity," Canad. J. Neuro. Sci., vol. 16, no. 2, pp. $180-183,1989$.

[16] C. Kirollos, A. Charlett, C. J. A. O’Neill, R. Kosik, K. Mozol, A. G. Purkiss, S. G. Bowes, P. W. Nicholson, W. B. Hunt, C. Weller, S. M. Dobbs, and R. J. Dobbs, "Objective measurement of activation of rigidity: Diagnostic, pathogenic and therapeutic implications on parkinsonism," Br. J. Clin. Pharmacol., vol. 41, no. 6, pp. 557-564, 1996.

[17] J. Ghika, A. W. Wiegner, J. J. Fang, L. Davies, R. R. Young, and J. H. Growdon, "Portable systems for quantifying motor abnormalities in Parkinson's disease," IEEE Trans. Biomed. Eng., vol. 40, pp. 276-283, Mar. 1993.

[18] A. W. Wiegner and R. L. Watts, "Elastic properties of muscles measured at the elbow in man-I: Normal controls," J. Neurol. Neurosurg. Psych., vol. 49, no. 10, pp. 1171-1176, 1986.

[19] M. Lakie, E. G. Walsh, and G. W. Wright, "Resonance at the wrist demonstrated by the use of a torque motor: An instrumental analysis of muscle tone in man," J. Physiol., vol. 353, pp. 265-285, Aug. 1984.

[20] M. P. Caligiuri and D. R. Galasko, "Quantifying drug-induced changes in parkinsonian rigidity using an instrumented measure of activated stiffness," Clin. Neuropharmacol., vol. 15, no. 1, pp. 1-12, 1992.
[21] M. P. Caligiuri, "Portable device for quantifying Parkinsonian wrist rigidity," Mov. Disord., vol. 9, no. 1, pp. 57-63, 1994.

[22] D. Halpern, R. Patterson, R. Mackie, W. Runck, and L. Eyler, "Muscular hypertonia: Quantitative analysis," Arch. Phys. Med. Rehabil., vol. 60, no. 5, pp. 208-218, 1979.

[23] S. K. Patrick, S. C. Naaman, M. J. A. Gauthier, and A. Prochazka, "A device for the quantification of Parkinsonian rigidity [abstract]," in Society for Neuroscience, 1995.

[24] S. K. Patrick, A. Denington, M. J. A. Gauthier, and A. Prochazka, "Sensing limb displacement to quantify limb stiffness [abstract]," in Society for Neuroscience, 1997.

[25] S. K. Patrick and A. Prochazka, "Testing of a device for the quantification of limb stiffness [abstract]," Can. J. Physiol. Pharmacol., vol. 74, p. Axxv, 1996.

[26] J. G. Snodgrass, G. Levy-Berger, and M. Haydon, Human Experimental Psychology. New York: Oxford Univ. Press, 1985.

[27] P. Martínez-Martín, A. Gil-Nagel, L. M. Gracia, B. Gómez, J. MartínezSarriés, F. Bermejo, and T. C. M. Group, "Unified Parkinson's disease rating scale characteristics and structure," Mov. Disord., vol. 9, no. 1, pp. 76-83, 1994

[28] C. C. Goetz, G. T. Stebbins, T. A. Chmura, S. Fahn, H. L. Klawans, and C. D. Marsden, "Teaching tape for the motor section of the unified Parkinson's disease rating scale," Mov. Disord., vol. 10, no. 3, pp. 263-266, 1995.

[29] J. W. Langston, H. Widner, C. G. Goetz, D. Brooks, S. Fahn, T. Freeman, and R. Watts, "Core assessment program for intracerebral transplantations (CAPIT)," Mov. Disord., vol. 7, no. 1, pp. 2-13, 1992.

[30] G. K. Montgomery, N. C. Reynolds, and R. M. Warren, "Qualitative assessment of Parkinson's disease: Study of reliability and data reduction with an abbreviated Columbia scale," Clin. Neuropharmacol., vol. 8, no. 1, pp. 83-92, 1985

Susan K. Patrick received the B.Sc. degree in general science, and the Ph.D. degree in neuroscience from the University of Alberta, Edmonton, AB, Canada, in 1992 and 1999 , respectively.

Her research involves development of methods to quantify tone in the upper limb in Parkinson's disease and hemiplegia, and application of these quantification methods to the clinical and surgical setting.

Allen A. Denington received the Dip. in electronics engineering technology from the Northern Alberta Institute of Technology, Edmonton, AB, Canada, in 1994

Since December 1994, he has been employed by the Division of Neuroscience at the University of Alberta. He is involved in the design, development, miniaturization, and production of medical electronic devices, laboratory electronics and mechanisms. His work includes the production of battery powered microprocessor controlled muscle stimulators.

Michel J. A. Gauthier (S'87-A'89) received the B.Sc. degree in mechanical engineering from the University of Alberta, Edmonton, $\mathrm{AB}$, Canada, and the Dip. computer engineering technology from Westerra Institute of Technology, Stony Plain, AB, Canada, in 1985, and 1989, respectively.

Since 1989, he has been with the Division of Neuroscience at the University of Alberta. His work includes the development of microprocessor controlled muscle stimulators, laboratory electronics and mechanisms, and software and firmware for analysis and control.

Deborah M. Gillard (S'91-M'92) received the B.Sc. and M.Sc. degrees in electrical engineering from the University of Alberta, Edmonton, AB, Canada, in 1991 and 1994, respectively.

Since 1995, she has been with the Division of Neuroscience at the University of Alberta as a Research Associate. She has been involved in the design, development, and testing of electrical stimulation devices to improve functional movement. Her areas of interest include system identification and adaptive control. 
Arthur Prochazka (M'74-SM'78) received the B. Eng. and the M.Sc. degrees from the University of Melbourne, Australia, and the Ph.D. degree from the University of Ulm, Germany, in 1967, 1970, and 1973, respectively.

After postdoctoral studies at Monash University, Melbourne, he moved to the U.K. in 1977 as a Lecturer of Physiology, St. Thomas's Hospital, University of London. In 1986, he was appointed Professor of Physiology at the University of Alberta, Edmonton, AB, Canada. His research has focused on the sensorimotor control of mammalian movement and, in recent years, the development of electronic devices to measure and improve movement in neurological disorders. 